\title{
Correction to: The trench warfare of gender discrimination: evidence from academic promotions to full professor in Italy
}

\author{
Giulio Marini $^{1}$ (D) Viviana Meschitti ${ }^{2}$ \\ Published online: 3 January 2019 \\ (c) Akadémiai Kiadó, Budapest, Hungary 2019
}

\section{Correction to: Scientometrics (2018) 115:989-1006 https://doi.org/10.1007/s11192-018-2696-8}

In the original publication of the article, the second sentence of the Abstract, one of the references and its corresponding citations were incorrectly published. The correct version is given in this Correction.

In Abstract, second sentence, "The new promotion system pursuant Gelmini Law (210/2010) in Italy implies to go through two steps." should read as "The new promotion system pursuant Gelmini Law (240/2010) in Italy implies to go through two steps."

In Introduction section, the fourth para, page 990, the sentence "Fresh literature shows that this new process is helping younger and productive candidates to progress quicker in their careers (Marini 2016)." should be replaced by "Fresh literature shows that this new process is helping younger and productive candidates to progress quicker in their careers (Marini 2017)."

In Discussion section, the first para, page 1001, the sentence "This paper provides a notable contribution when considered together with previous studies looking at the achievement of ASN (Bagues et al. 2017; Marini 2016; Marzolla 2016):" should be replaced by "This paper provides a notable contribution when considered together with previous studies looking at the achievement of ASN (Bagues et al. 2017; Marini 2017; Marzolla 2016):"

The reference Marini, G. (2016) in the original publication should read as below:

Marini, G. (2017). New promotion patterns in Italian universities: Less seniority and more productivity? Data from ASN. Higher Education, 73(2), 189-205. https://doi. org/10.1007/s10734-016-0008-x.

The original article can be found online at https://doi.org/10.1007/s11192-018-2696-8.

Giulio Marini

g.marini@ucl.ac.uk

Viviana Meschitti

v.meschitti@hud.ac.uk

1 Centre for Global Higher Education, University College London, Institute of Education, 20 Bedford Way, London WC1H 0A, UK

2 Huddersfield Business School, University of Huddersfield, Queensgate, Huddersfield HD1 3DH, UK 